Gynäkologe 2020 · 53:414-415

https://doi.org/10.1007/s00129-020-04625-z

(c) Springer Medizin Verlag GmbH, ein Teil von Springer Nature 2020

\author{
Klaus Vetter $\cdot$ Roland Zimmermann ${ }^{2}$ \\ 'Berlin, Deutschland \\ ${ }^{2}$ Klinik für Geburtshilfe, Universitätsspital Zürich, Zürich, Schweiz
}

\title{
Translation - Von Konzepten der intrauterinen Entwicklung zu therapeutischen Konzepten bei schwerer plazentarer Pathologie
}

den kann, ja muss, weist Thorsten Braun in seinem Artikel „Intrauterine Prägung - Das Konzept und die Bedeutung der Plazenta“ nach.

\section{》) Forschung zu pränatalen Risiken für Kognition, Motorik, Resilienz u. a. kann Prävention optimieren}

Mangelentwicklung bewirken. Dies sind kurzfristige, während der Schwangerschaft bzw. nach der Geburt feststellbare Konsequenzen, die lange bekannt sind.

Die Frage des vorliegenden Hefts ist aber, welche langfristigen Auswirkungen Einflüsse in der Schwangerschaft auf das Leben des Ungeborenen haben können, welche Mechanismen dies bewirken können und, nicht zuletzt, wie sich mögliche unerwünschte Folgen vermeiden lassen.

Die Plazenta kann als vermittelndes Organ angesehen werden, das im hier vorgestellten Modell epigenetisch verändert werden kann mit Folgen für ihre Morphologie, Transportsysteme und endokrine Funktionen. Damit kann eine Reihe Folgen antenataler Glukokortikoidgaben abgeleitet werden, die einerseits kurzfristig als Lungenreifeinduktion eingesetzt werden, andererseits vermindertes Wachstum bedingen. Sie beeinflussen aber auch endokrine Steuerungsvorgänge zwischen Hypothalamus, Hypophyse und Nebennieren und damit nicht zuletzt die spätere endokrine Stressreaktivität des Ungeborenen. Dass dies alles als ein geschlechtsabhängig komplexes $\mathrm{Ge}$ schehen an der Plazenta angesehen wer-
$\mathrm{Zu}$ den interessanten Angriffspunkten für bleibende Veränderungen im Sinn einer fetalen Programmierung gehören die Telomere. Ihre Biologie könnte als Mechanismus verstanden werden, wie ein erhöhtes Krankheitsrisiko durch intrauterine Einflussfaktoren auf den heranwachsenden Organismus vermittelt wird. Sonja Entringer und Claudia Lazarides diskutieren in ihrem Artikel „Fetale Programmierung von Zellalterungsprozessen“ Studienergebnisse, aus denen hervorgeht, dass u. a. ungünstige Ernährung, psychosozialer Stress und andere medizinische Risiken, wie Mangelversorgung, während der Schwangerschaft mit verkürzten Telomeren der Feten einhergehen. Es ist bekannt, dass kürzere Telomere mit einem erhöhten Risiko für altersbedingte Erkrankungen und kürzere Lebensdauer assoziiert sind. Positive Effekte werden u.a. mit Mind-Body-Interventionen in Zusammenhang gebracht. Ziel ist es, auf Basis dieser Erkenntnisse Risiken für den Fetus frühestmöglich zu identifizieren und dies sowohl für eine maximale Wirksamkeit präventiver Interventionen einzusetzen als auch dafür, eine mögliche fetale Programmierung von Krankheitsrisiken zu verhindern bzw. eventuell sogar wieder rückgängig zu machen.

Dieser Ansatz stellt den Boden der Arbeit von Birgit Arabin und Gerlinde A.S. Metz dar: „Environmental Enrichment und Schwangerschaft - Paradigmen (epi)genetischer Folgen sozialer oder sensueller Stimulation“. Beide greifen dies auf, indem sie davon ausgehen, dass Stressfaktoren in der Schwangerschaft auf die kognitive Entwicklung des Fetus und damit auf sein gesamtes Leben Einfluss nehmen können. Auf Grundlage der pränatalen Sinnesentwicklung werden Angriffspunkte für positive, nichtinvasive Interventionen herausgearbeitet, um die Resilienz des Feten schon intrauterin positiv zu beeinflussen. Langfristig ist eine erhöhte Telomerlänge das Ziel der Interventionen, und damit Gesundheit und ein langes Leben. Sie umfassen u. a. kreatives Schreiben, Modellieren, Bewegung zu Musik oder Singen. Entsprechende prospektive Studien sind schon im Gang.

Die letzte Arbeit zu langfristigen Auswirkungen eines ungünstigen intrauterinen Milieus beruht auf manifesten Störungen der nutritiven und respiratorischen Plazentafunktion. Sie sind definiert durch dopplersonographisch erfasste schwer beeinträchtigte Blutströmung (ARED[,absent or reversed enddiastolic flow"]-Flow) in den Aa. umbilicales. Karel Maršál und Klaus Vetter äußern sich aus klinischer Sicht zu „Kon- 
sequenzen aus intrauterin erkannter plazentarer Versorgungsstörung“. Am Beispiel langfristiger neurokognitiver und kardiovaskulärer negativer Folgen einer nachgewiesenen eingeschränkten fetoplazentaren Blutströmung wird die Bedeutung der beeinträchtigten Versorgung verdeutlicht. Pathophysiologische Zusammenhänge werden als Grundlage nützlicher, z.T. aber auch fatal schädigender Anpassungsvorgänge herausgearbeitet, um schließlich eine vorgezogene Geburt - noch vor dem Auftreten klassischer Entscheidungsaspekte, wie CTG(Kardiotokographie)oder Kreislaufveränderungen beim $\mathrm{Fe}$ tus - vorzuschlagen. Dies alles wird durch neueste Studien belegt, die solche Entscheidungen auch schon am Rand der Lebensfähigkeit begründen.

Translation - in allen 4 Artikeln finden sich Handlungshinweise auf Basis möglicher ungünstiger Umstände, so

- 1. Überlegungen zum reduzierten

Einsatz pränataler Glukokortikoide,

- 2. Schaffen eines Milieus, das eine

Verkürzung von Telomeren vermeidet, im besten Fall sogar zu ihrer Verlängerung beiträgt,

- 3. eine externe positive Stimulation von Sinnesorganen des Fetus zur Festigung seiner Resilienz im späteren Leben und,

- 4., nicht zuletzt, eine rechtzeitige Geburt - ehe mögliche Schäden durch Mangelernährung oder Hypoxie erkannt werden und sich manifestieren können.
Diese 4 exemplarischen Arbeiten weisen nach, dass Pränatalmedizin und Geburtshilfe sich im Sinn zukünftiger Generationen weiterentwickeln wird und dass es sich lohnt, auch ohne externe wirtschaftliche Interessen akademisch in die Schwangerschaft $\mathrm{zu}$ investieren.

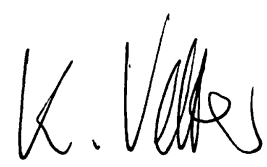

Klaus Vetter

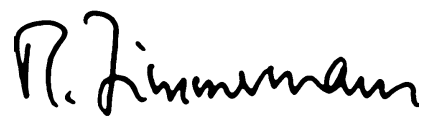

\section{Roland Zimmermann}

\section{Korrespondenzadresse}

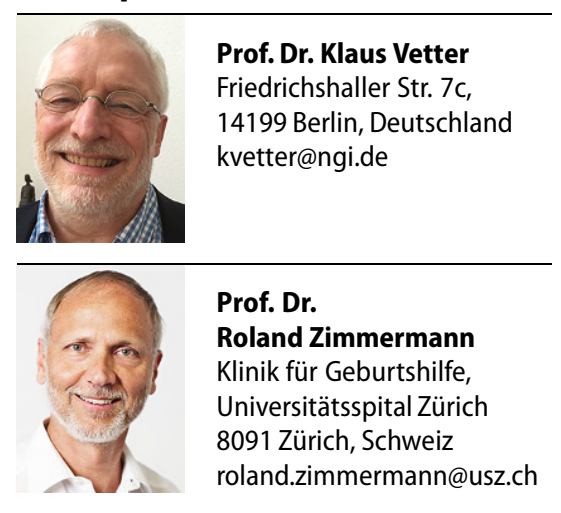

Interessenkonflikt. K. Vetter und R. Zimmermann geben an, dass kein Interessenkonflikt besteht.
Gaisser, Andrea, Weg-Remers, Susanne Patientenzentrierte Information in der onkologischen Versorgung

Evidenz und mehr

Berlin/Heidelberg: Springer-Verlag GmbH 2020, 1. Auflage, (ISBN: 978-3662-60460-1), 44,99 EUR

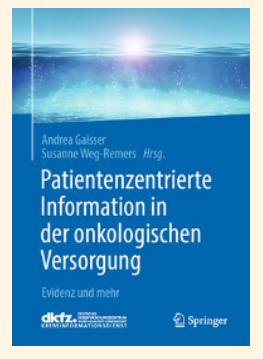

In Zusammenarbeit mit dem Krebsinformationsdienst ist im SpringerVerlag ein neues Buch erschienen. Es richtet sich an Ärztinnen

und Ärzte sowie Angehörige aller Gesundheitsberufe, die an der Versorgung von Krebspatienten beteiligt sind.

Das Buch „Patientenzentrierte Information in der onkologischen Versorgung" fasst die über Jahrzehnte gesammelten Erfahrungen des Krebsinformationsdienstes des Deutschen Krebsforschungszentrums im Umgang mit individuellen Anfragen von Krebspatienten zusammen und bietet Unterstützung beim Beantworten typischer und manchmal auch schwieriger Fragen zu den für Patienten wichtigen Themen.

Die Buchbeiträge folgen den Fragen, die sich viele Patientinnen und Patienten auf ihrem Weg durch die Krebserkrankung stellen. Illustriert anhand beispielhafter Anfragen werden ihre Bedeutung und praktische Relevanz für Patienten ebenso dargestellt wie die jeweilige gesicherte Datenlage. Zu jedem Kapitel sind weiterführende Informationen, auch für Patienten, und eine Literaturauswahl zusammengestellt. Eine Einführung zu den Herausforderungen der Informationsvermittlung und Kommunikation in der Onkologie sowie eine Auswahl nützlicher Hinweise und Empfehlungen für Informationssuche, Informationsbewertung und Kommunikation runden den Inhalt des Buches ab. 